\title{
Polymer dynamics in a model of the turbulent buffer layer
}

\author{
Philip A. Stone and Michael D. Graham a) \\ Department of Chemical Engineering, University of Wisconsin-Madison, Madison, Wisconsin 53706-1691
}

(Received 12 September 2002; accepted 30 January 2003; published 3 April 2003)

\begin{abstract}
A Brownian dynamics study of bead-spring-chain polymer dynamics is undertaken in a model flow that captures key features of the buffer region of near-wall turbulence-wavy streamwise vortices superimposed on a mean shear. In this flow and in any Lagrangian chaotic flow, a Hookean dumbbell polymer will stretch indefinitely if and only if the Weissenberg number based on the largest Lyapunov exponent for the velocity field is $\geqslant \frac{1}{2}$. In the flow investigated here, this criterion is found to be good predictor of when the stretch of finitely extensible chains approaches its maximum value. The chains become highly stretched in the streamwise streaks and relax as they move into and around the vortex cores, leading to large differences in stress in different regions of the flow. Hydrodynamic and excluded volume interactions between polymer segments have no qualitative effects once results are normalized for the change in relaxation time due to their inclusion. The results from the bead-spring-chain models are used to assess the utility of the simpler FENE-P model. Although the FENE-P model does not capture the hysteresis in stress that is seen with the bead-spring-chain models, it otherwise qualitatively captures the behavior of the bead-spring chains. Most importantly, large polymer stress in the flow is seen at the same spatial positions for both the FENE-P and the more detailed models. (C) 2003 American Institute of Physics. [DOI: 10.1063/1.1563258]
\end{abstract}

\section{INTRODUCTION}

The study of polymer drag reduction, the reduction of skin friction in turbulent flow by the addition of polymers ${ }^{1-3}$ is hindered by the fact that the exact behavior of polymers in turbulent flows is not known. Experimental procedures have yet to be developed that probe the extension of macromolecules in turbulent flows. To gain a better understanding of how real polymers behave in turbulent flows, we have undertaken a computational study using Brownian dynamics (BD) simulations of bead-spring-chain polymer models with a large number of internal time scales in an "exact" model flow, described below, that captures the coherent structures of the turbulent buffer region. We focus on the buffer region because it is where the strain rates are largest, is the most important in terms of production and dissipation of turbulent energy, ${ }^{4}$ and is known to be modified in dragreducing flows. ${ }^{5-9}$ The bead-spring models allow us to determine the effects of the properties of real polymers (internal time scales, finite extensibility, hydrodynamic interactions, excluded volume) on extension and stress in turbulent flows.

In this study, the concentration of polymers is assumed to be so small that the effect of the polymer on the flow field is negligible. In a related study, ${ }^{10,11}$ we have used the simple FENE-P model ${ }^{12}$ in fully coupled simulations, to determine how polymers affect the structures of the buffer region. The BD simulations presented here are thus intended not only to illustrate how polymers behave in the coherent structures of the turbulent buffer region but also to assess the utility of the

${ }^{a)}$ Electronic mail: graham @engr.wisc.edu relatively simple FENE-P dumbbell model in coupled simulations of turbulent flows of polymer solutions.

A key structural observation from experiments and direct numerical simulations (DNS) of drag-reducing polymer solutions is the modification of the buffer layer structures. ${ }^{5-9,13-15}$ The dominant structures of the buffer layer are pairs of counter-rotating, streamwise vortices that lead to streaks in the streamwise velocity. ${ }^{4}$ The streaks in a dragreducing flow are known to be wider than in the Newtonian case $^{6,14}$ and the streamwise enstrophy, ${ }^{16}$ which measures the strength of the streamwise vortices, is reduced. ${ }^{13}$ Since the near-wall buffer region is the most important in the production and dissipation of turbulent energy ${ }^{4}$ and is known to be modified in drag reduction, it is clear that any effort to mechanistically understand rheological drag reduction should address this region. Ideally, we would like to study polymers in a nonturbulent flow that still captures the flow structures characteristic of this region. Fortunately, such flows exist in the recently discovered "exact coherent states" (ECS) that arise in plane shear flows.

The $\mathrm{ECS}^{17-23}$ are a family of three-dimensional, steady (in a traveling reference frame) solutions to the NavierStokes equation that appear at via saddle-node bifurcations $^{24,25}$ at Reynolds numbers Re near the transition value seen in experiments. ${ }^{26,27}$ These states were first found in plane Couette flow, ${ }^{17-21}$ but have also been shown to exist in plane Poiseuille flow. ${ }^{22,23}$ They consist of a mean shear flow with superimposed wavy streamwise vortices and the associated high- and low-speed streaks in the streamwise velocity. They are periodic in the streamwise $(x)$ and spanwise $(z)$ directions, with wavelengths $l_{x}$ and $l_{z}$, respectively. Recent parametric studies by Waleffe ${ }^{28}$ of the ECS in Poiseuille 


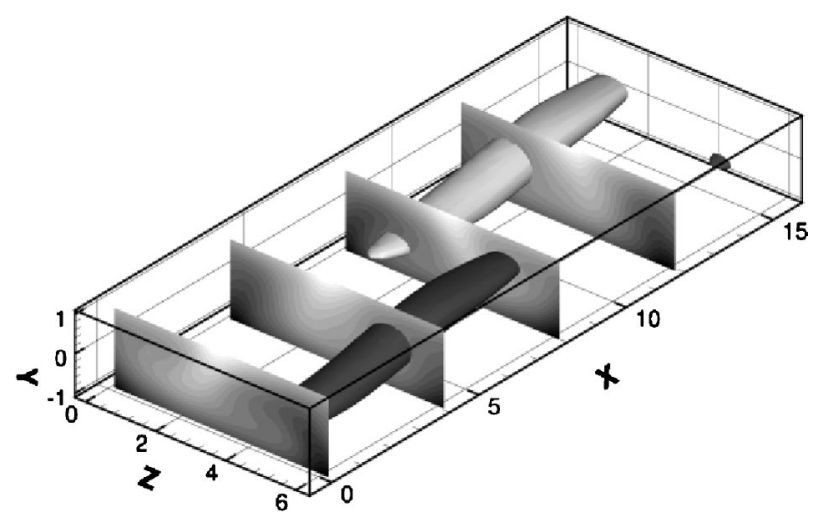

FIG. 1. "Exact coherent state" in plane Couette flow. The contours are of $v_{x}$ (white is positive, black is negative). The constant vorticity surfaces are $\omega_{x}=-0.36 U / L$ (dark gray) and $\omega_{x}=0.36 U / L$ (light gray). $U$ and $L$ are the characteristic velocity and half-channel height.

flow found that the optimal spanwise wavelength of these (the wavelength at which the onset Reynolds number is lowest) is about 100 wall units, in quantitative agreement with the streak spacing observed in the turbulent buffer layer. In the present study we use a variant of Couette flow with constant vorticity (shear rate) boundary conditions at the wall. These boundary conditions eliminate the viscous sublayer, the thin region closest to the wall that does not have a large role in turbulence production and dissipation.

Figure 1 shows contours of streamwise velocity, $v_{x}$, for an ECS at various $x$ values for $\mathrm{Re}=110, l_{x}=2 \pi / 0.4, l_{z}$ $=2 \pi$. Here lengths are scaled with the the channel halfheight $L$; the wall shear rate is fixed at $U / L$ where $\pm U$ are the streamwise velocities at the top and bottom in the trivial Couette flow state (linear velocity profile); and $\operatorname{Re}=U L / \nu$ where $\nu$ is the kinematic velocity. In wall units, the streamwise and spanwise wavelengths are $l_{x}^{+}=165$ and $l_{z}^{+}=66$, respectively. The wavy high speed streamwise streak is evident in white. The surfaces of Fig. 1 are of constant streamwise vorticity, $\omega_{x}$, with the lighter gray being positive vorticity and darker gray being negative vorticity. These are the counter-rotating vortices that flank the streak. This structure is similar to that of the buffer region. ${ }^{22}$

The mechanism underlying the ECS is reasonably well understood. ${ }^{21}$ A perturbation in the form of streamwise vortices redistributes the mean shear leading to a spanwise inflectional stratification of streamwise velocity. The boundary between fluids of differing velocities undergoes a KelvinHelmholtz instability that concentrates wall-normal vorticity. This vorticity component is then tilted into the streamwise direction and stretched by the mean shear. This tilting and stretching of wall-normal vorticity reinforces the streamwise vortices and the process is thereby self-sustained. From the dynamical systems point of view, these states appear to form a part of the dynamical skeleton of the turbulent flow: i.e., they are saddle points that underlie, in part, the strange attractor of turbulence. ${ }^{29,30}$ In short, despite their relative simplicity, the ECS seem to capture important features of the buffer layer turbulence: we believe, therefore, that they provide an excellent model system for studying the interaction of rheology and turbulence.
This view is supported by our studies of how the Couette flow ECS are affected by viscoelasticity in the form of the FENE-P constitutive model. ${ }^{11}$ All of the effects that we see are consistent with experimental and DNS observations of drag reducing solutions. The addition of polymer stress to the ECS causes an increase in the Reynolds number at which the ECS appear. Compared to the Newtonian ECS, the viscoelastic exact coherent states show a decrease in the "waviness" of the streamwise streak, a decrease in the wall-normal velocity fluctuations, an increase in the streamwise velocity fluctuations, an increase in the fluctuation kinetic energy, and an increase in the average streamwise velocity-drag reduction occurs. In the ECS, the polymer stretches in the streamwise streaks and relaxes as it goes into and around the vortices. This relaxation of the polymer produces forces in the wall-normal and streamwise directions that work against the vortices, reducing their strength. This effect is reflected in the divergence of the polymer stress tensor, which shows a large force in the negative $x$ (streamwise) direction where the polymer is slowing and leaving the streak and entering a vortex. This force acts directly to reduce the $x$ velocity in the areas of interconnection of the streaks and vortices and straightens the streaks. Since the self-sustaining process of the ECS depends on the vortices to redistribute mean shear, the decrease in their strength leads to a collapse of the process and, therefore, drag reduction. By examining the behavior of more realistic polymer models in the ECS flows, the present work provides data to allow an assessment of the robustness of conclusions drawn from the coupled simulations with simple polymer models.

\section{POLYMERS IN LAGRANGIAN TIME-DEPENDENT FLOWS}

In a steady elongational flow with extension rate $\dot{\epsilon}$, a Hookean dumbbell model of a polymer chain will extend indefinitely if the Weissenberg number $\lambda \dot{\epsilon}>\frac{1}{2}$, where $\lambda$ is the stress relaxation time for the dumbbell. The large stress, or equivalently, extensional viscosity, arising from this large degree of extension has long been recognized as important in the drag reduction phenomenon. Lumley derived an approximate result for turbulent flow that shows that a polymer molecule becomes highly stretched once the turbulent strain rate exceeds a certain threshold ${ }^{31}$ (see also Refs. 32-34). Massah and co-workers performed simulations of a FENE beadspring chain in a direct numerical simulation of turbulent channel flow and found that in the buffer region the extension and orientation of the polymer fluctuate with time, and the transient polymer stretch is significantly larger than its coiled equilibrium length. ${ }^{35}$ This result is only seen when the product of the polymer relaxation time and the characteristic wall shear rate (a nominal Weissenberg number We) exceeds a certain threshold. Later work by the same group found that the stresses introduced by the stretched polymers in a turbulent flow lead to added dissipations, both positive and negative. ${ }^{36}$ The positive dissipations are associated with the streamwise vortices in the near-wall region and would decrease the production of Reynolds shear stress in these structures. A study of FENE dumbbells in steady, Gaussian ran- 
dom flows shows that stochastic flow fields can produce large conformation changes in the polymer. ${ }^{37}$ Again, the conformation change occurs above a critical nominal Weissenberg number. Recent work by Ilg et $_{\text {al. }}{ }^{38}$ studied the dynamics of FENE and FENE-P dumbbells in wall turbulent flow. The flow field was taken from a fully coupled DNS of a drag-reducing (FENE-P) polymer solution. They find that polymers can become highly stretched in near-wall turbulence and highly oriented parallel to the mean shear. A comparison of the Lagrangian averages of the FENE and FENE-P dumbbell show that the extensions are qualitatively similar with the FENE-P model giving slightly greater extension when velocity gradients are high and polymers are highly stretched. The stretching of an ensemble of FENE dumbbells integrated along the same trajectory in the flow is characterized by a broad (instantaneous) distribution of polymer extensions. We now present a result that unifies the above-mentioned studies, providing a specific and general criterion for the onset of large polymer stress during flow.

Consider a flow that is unbounded or spatially periodic in at least one direction, so that it makes sense to consider the behavior along a fluid trajectory as $t \rightarrow \infty$. The end-to-end vector, q, of a Hookean dumbbell polymer model evolves in the flow field, $\mathbf{v}$, according to the Ito stochastic differential equation,

$$
d \mathbf{q}=\left(\boldsymbol{\kappa} \cdot \mathbf{q}-\frac{1}{2 \lambda} \mathbf{q}\right) d t+\sqrt{\frac{4 k_{\mathrm{B}} T}{\zeta}} d \mathbf{w},
$$

where $\boldsymbol{\kappa}=(\boldsymbol{\nabla} \mathbf{v})^{T}, k_{\mathrm{B}}, T$, and $\zeta$ are Boltzmann's constant, temperature and the bead friction coefficient, and $d \mathbf{w}$ is a vector of differentials of independent Wiener processes. If the relaxation and Brownian terms in this equation are dropped, this is simply the evolution equation for an infinitesimal material line element. With initial condition $\mathbf{q}=\mathbf{q}_{0}$, Eq. (1) has the solution ${ }^{39}$

$$
\begin{aligned}
\mathbf{q}(t)= & e^{-t / 2 \lambda} \mathbf{E}\left(\mathbf{x}_{0}, t, 0\right) \cdot \mathbf{q}_{0} \\
& +\sqrt{\frac{4 k_{\mathrm{B}} T}{\zeta}} \int_{0}^{t} e^{\left(t^{\prime}-t\right) / 2 \lambda} \mathbf{E}\left(\mathbf{x}_{0}, t, t^{\prime}\right) \cdot d \mathbf{w}\left(t^{\prime}\right),
\end{aligned}
$$

where $\mathbf{E}\left(\mathbf{x}_{0}, t, t^{\prime}\right)$ is the displacement gradient tensor ${ }^{40}$ and $\mathbf{x}_{0}$ is the initial position of the dumbbell in the velocity field. Taking the ensemble average of this, on the condition that $\mathbf{q}(0)=\mathbf{q}_{0}$, we arrive at

$$
\left\langle\mathbf{q}(t) \mid \mathbf{q}_{0}\right\rangle=e^{-t / 2 \lambda} \mathbf{E}\left(\mathbf{x}_{0}, t, 0\right) \cdot \mathbf{q}_{0},
$$

because $\langle d \mathbf{w}(t)\rangle=0$. Now one can define a set of "polymer Lyapunov exponents," measuring the time-average rate of polymer stretch of a dumbbell, as

$$
\begin{aligned}
\sigma_{p} & =\lim _{t \rightarrow \infty} \frac{1}{t} \log \frac{\left\|\left\langle\mathbf{q}(t) \mid \mathbf{q}_{0}\right\rangle\right\|}{\left\|\mathbf{q}_{0}\right\|} \\
& =\lim _{t \rightarrow \infty} \frac{1}{t} \log \frac{e^{-t / 2 \lambda}\left\|\mathbf{E}\left(\mathbf{x}_{0}, t, 0\right) \cdot \mathbf{q}_{0}\right\|}{\left\|\mathbf{q}_{0}\right\|} .
\end{aligned}
$$

The Lyapunov exponents $\sigma$ for the velocity field-the Lagrangian time-averaged material line stretch rates-satisfy the same expression, but with $1 / \lambda$ set to zero. In fact, for randomly chosen $\mathbf{q}_{0}$, this formula gives the largest Lyapunov exponent. So the polymer Lyapunov exponents are related to the flow Lyapunov exponents by the simple relationship

$$
\sigma_{p}=\sigma-\frac{1}{2 \lambda} .
$$

For $\sigma_{p}>0$, the polymer stretches at an exponential rate. If $\sigma_{\max }$ defines the largest Lyapunov for the flow field, Hookean dumbbells will stretch indefinitely in a flow if and only if

$$
\mathrm{We}_{\sigma}=\sigma_{\max } \lambda>\frac{1}{2},
$$

where $\mathrm{We}_{\sigma}$ is a Weissenberg number based on $\sigma_{\max }$. This result is quite similar to the result of Lumley ${ }^{30}$ and is a starting point for recent theoretical work on the dynamics of passive polymer molecules in random flows. ${ }^{32,33,41}$ All flows with chaotic particle paths, including turbulent flows ${ }^{42}$ and the ECS flows, ${ }^{10,11}$ have a positive $\sigma_{\max }$, as does the Gaussian random flow studied in Ref. 37. Our coupled study of the effect of FENE-P dumbbells on the ECS shows that the polymer begins to strongly affect the flow structure when $\mathrm{We}_{\sigma}$ $\approx 1 / 2$. One of our goals in the present work is to see how well this criterion applies to more general models of dilute polymer solutions.

\section{POLYMER MODELS AND SIMULATION TECHNIQUES}

The BD simulation that we use in this work was developed by Jendrejack, de Pablo, and Graham. ${ }^{43,44}$ The polymer is modeled as beads, where the mass of the molecule is concentrated, connected by finitely extensible springs, which represent the entropic restoring force in the polymer. The model contains $N$ beads connected by $N-1$ springs. Ignoring the inertia of the beads, the equation of motion for a bead of the chain can be found by balancing all the forces acting on the bead: ${ }^{12}$

$$
\mathbf{F}_{\nu}^{(h)}+\mathbf{F}_{\nu}^{(b)}+\mathbf{F}_{\nu}^{(\phi)}=\mathbf{0} \quad(\nu=1, \ldots, N) .
$$

The beads experience three kinds of forces.

(a) A hydrodynamic drag force $\mathbf{F}_{v}^{(h)}$. This force is the resistance that the bead experiences as it moves through the solvent. Stokes' law is used to find the drag and, assuming that the hydrodynamic drag is isotropic, gives

$$
\mathbf{F}_{\nu}^{(h)}=-\zeta\left(\dot{\mathbf{r}}_{\nu}-\left(\mathbf{v}_{\nu}+\mathbf{v}_{\nu}^{\prime}\right)\right),
$$

where $\mathbf{r}_{\nu}$ is the position of bead $\nu, \zeta$ is the friction coefficient, $\mathbf{v}_{\nu}=\mathbf{v}_{0}+\left[(\boldsymbol{\nabla} \mathbf{v})^{T} \cdot \mathbf{r}_{\nu}\right]$ is the imposed velocity at bead $\nu$, and $\mathbf{v}_{\nu}^{\prime}$ is the perturbation of the velocity field at bead $\nu$ due to the motion of the other beads in the chain. This perturbation is referred to as "hydrodynamic interaction" or HI. The addition of $\mathrm{HI}$ to the model causes its largest relaxation time to decrease compared to a model without HI (a "freedraining" model), due to the cooperative motion among the beads, which speeds up the relaxation to equilibrium.

(b) A Brownian force $\mathbf{F}_{v}^{(b)}$. A large particle, relative to the size of the solvent particles, in a solution experiences a rapidly fluctuating force due to the thermal fluctuations of the solvent. This force is assumed to vary extremely rapidly 
compared to fluctuations in the bead velocity. The Brownian force is related to the hydrodynamic drag force through the fluctuation-dissipation theorem. ${ }^{39}$

(c) An intramolecular force $\mathbf{F}_{\nu}^{(\phi)}$. This includes the force on the bead due to the springs connecting it to other beads. For this study, we will exclusively use the finitely extensible $n$ onlinear elastic (FENE) spring with spring force, $\mathbf{F}^{(c)}$, given by

$$
\mathbf{F}^{(c)}=\frac{H \mathbf{Q}}{1-\left(Q / Q_{0}\right)^{2}}, \quad Q \leqslant Q_{0},
$$

where $\mathbf{Q}$ is the "connector vector" that connects pairs of adjacent beads, $H$ is the Hookean spring constant, and $Q_{0}$ is the maximum extension of the spring. Since $Q_{0}$ is the contour length of the segment of the polymer represented by the spring, $Q_{0}=b_{k} N_{k, s}$, where $b_{k}$ is the Kuhn length and $N_{k, s}$ is the number of Kuhn lengths per spring. The intramolecular force also includes the excluded volume (EV) potential. EV, which represents interactions between segments far apart along the polymer chain, causes the equilibrium size of the polymer to increase. ${ }^{45}$ This increase in equilibrium size causes a slight increase in the largest relaxation time of the polymer. For the current work, the excluded volume potential between two beads on a chain is given by ${ }^{43}$

$$
U_{i j}^{\mathrm{EV}}=\frac{1}{2} v k_{\mathrm{B}} T N_{k, s}^{2}\left(\frac{3}{4 \pi S_{s}^{2}}\right)^{3 / 2} \exp \left(\frac{-3 R_{i j}^{2}}{4 S_{s}^{2}}\right),
$$

where $v$ is the excluded volume parameter, $k_{\mathrm{B}}$ is the Boltzmann constant, $T$ is the absolute temperature, $R_{i j}$ is the distance between beads $i$ and $j$, and $S_{s}^{2}=N_{k, s} b_{k}^{2} / 6$. The hydrodynamic radius, $a$, for a segment of the chain is

$$
a=a^{\prime}\left(\frac{N_{k, s}}{6}\right)^{1 / 2},
$$

where $a$ is in units of Kuhn lengths. The value for $a^{\prime}$ in Eq. (11) is set to 0.64 , which comes from renormalization group theory. ${ }^{43,45}$ The excluded volume potential comes from considering the overlap of two Gaussian coils ${ }^{43,45}$ and its magnitude depends on the excluded volume parameter, $v$, which has units of Kuhn length cubed.

From the force balance above, one can derive a governing stochastic differential equation in the $3 \mathrm{~N}$ spatial coordinates for an isolated bead-spring chain in a thermal solvent: ${ }^{43}$

$$
\begin{aligned}
& d \mathbf{R}=\left(\mathbf{K} \cdot \mathbf{R}+\mathbf{D} \cdot \mathbf{F}+\frac{\partial}{\partial \mathbf{R}} \cdot \mathbf{D}\right) d t+\sqrt{2} \mathbf{B} \cdot d \mathbf{w}, \\
& \mathbf{D}=\mathbf{B} \cdot \mathbf{B}^{T} .
\end{aligned}
$$

Here $\mathbf{R}$ is the vector of the $3 N$ spatial coordinates of the beads of the chain, $\mathbf{D}$ is a $3 N \times 3 N$ diffusion tensor, $\mathbf{F}$ is a force vector acting on the chain, and $\mathbf{K}$ is a $3 N \times 3 N$ block diagonal matrix with diagonal components $\boldsymbol{\kappa}=(\boldsymbol{\nabla} \mathbf{v})^{T}$. The velocity gradient, $\boldsymbol{\kappa}$, is evaluated along a fluid trajectory in the ECS, so it is fluctuating in time. The hydrodynamic interactions enter Eq. (12) through the diffusion tensor, D. For this work we take D to be the Rotne-Prager-Yamakawa (RPY) tensor, ${ }^{46}$ which has the form

$$
\begin{aligned}
\mathbf{D}_{i i}= & \frac{k_{\mathrm{B}} T}{\zeta} \mathbf{I}, \\
\mathbf{D}_{i j}= & \frac{k_{\mathrm{B}} T}{8 \pi \eta R_{i j}}\left(C_{1} \mathbf{I}+C_{2} \frac{\mathbf{R}_{i j} \mathbf{R}_{i j}}{R_{i j}^{2}}\right), \\
& \text { if } i \neq j \quad \text { and } R_{i j} \geqslant 2 a, \\
\mathbf{D}_{i j}= & \frac{k_{\mathrm{B}} T}{\zeta}\left[\left(1-\frac{9 R_{i j}}{32 a}\right) \mathbf{I}+\frac{3}{32} \frac{\mathbf{R}_{i j} \mathbf{R}_{i j}}{a R_{i j}}\right], \\
& \text { if } i \neq j \quad \text { and } R_{i j}<2 a, \\
C_{1}= & 1+\frac{2 a^{2}}{3 R_{i j}^{2}}, \\
C_{2}= & 1-\frac{2 a^{2}}{R_{i j}^{2}},
\end{aligned}
$$

where $\mathbf{R}_{i j} \equiv \mathbf{R}_{j}-\mathbf{R}_{i}, R_{i j} \equiv \sqrt{\mathbf{R}_{i j} \cdot \mathbf{R}_{i j}}$, where the vector $\mathbf{R}_{i}$ contains the three Cartesian coordinates of the position vector of the $i$ th bead, and $\mathbf{I}$ is the $3 \times 3$ identity matrix. The Brownian displacement is $\sqrt{2} \mathbf{B} \cdot d \mathbf{w}$, where the vector $d \mathbf{w}$ has components obtained from a real-valued Gaussian distribution with mean zero and variance $d t$; Eq. (13) arises from the fluctuation-dissipation theorem. In some simulations, we neglect hydrodynamic interactions by setting $\mathbf{D}_{i j}=\mathbf{0}$ for $i \neq j$, giving a "free-draining" (FD) model.

The force $\mathbf{F}$ appearing in Eq. (12) is

$$
\mathbf{F}_{i}=-\frac{\partial}{\partial \mathbf{R}_{i}}\left(\sum_{|j-k|=1}-\frac{1}{2} b_{s} \ln \left[1-\frac{R_{j k}^{2}}{b_{s}}\right]+\sum_{j<k} U_{j k}^{\mathrm{EV}}\right),
$$

where $b_{s}=H Q_{0}^{2} / k_{\mathrm{B}} T=3 N_{k, s}$ is the square of the maximum dimensionless bond length. The first term in parentheses is the total potential energy for the FENE springs. The polymer contribution to the stress tensor, $\boldsymbol{\tau}_{p}$, is given by

$$
\boldsymbol{\tau}_{p}=n \sum_{i=1}^{N}\left\langle\left(\mathbf{R}_{i}-\mathbf{r}_{c}\right) \mathbf{F}_{i}\right\rangle+n N_{s} k_{\mathrm{B}} T \mathbf{I},
$$

where $n$ is the number density of molecules and $\mathbf{r}_{c}$ is the center of mass of the chain. ${ }^{43}$ In this paper, polymer stresses are scaled by $n k_{\mathrm{B}} T$.

In the current work, the stochastic differential equation [Eq. (12)] is solved for chains of 20 springs. Ensembles of 50 stochastic trajectories were typically used-this relatively small ensemble size is adequate because at the high Weissenberg numbers of interest here the distribution functions are quite narrow. For most of the simulations, the contour length of the chain is chosen to be that of $6 \times 10^{6}$ molecular weight polyethylene oxide (PEO) in water. This specification requires a chain of 39100 Kuhn segments, giving approximately 2000 Kuhn lengths per spring. Because computational results where the polymer stress is coupled to the flow field are only attainable for much smaller contour lengths (see, for example, Refs. 10 and 14), smaller polymer chains $(b=600)$ are also considered. We consider two values of $v$ in order to assess the effect of excluded volume. These values have not been matched to any particular polymer/solvent system; we shall see that once relaxation time has been nor- 
TABLE I. Relaxation times for the various polymer models. Models 1 and 4 are free-draining. Here $\lambda_{\text {Rouse }}$ is the characteristic relaxation time for a chain with no HI or EV and Hookean springs.

\begin{tabular}{rrrcccr}
\hline \hline & $N_{s}$ & $N_{k, s}$ & $a^{\prime}$ & $v$ & $\lambda / \lambda_{\text {Rouse }}$ & \multicolumn{1}{c}{$b$} \\
\hline 1 & 20 & 1955 & $\ldots$ & $\ldots$ & 0.8731 & 117300 \\
2 & 20 & 1955 & 0.64 & 1.0 & 0.7091 & 117300 \\
3 & 20 & 1955 & 0.64 & 20.0 & 0.7356 & 117300 \\
4 & 20 & 10 & $\ldots$ & $\cdots$ & 0.7334 & 600 \\
5 & 20 & 10 & 0.64 & 1.0 & 0.4673 & 600 \\
\hline \hline
\end{tabular}

malized, EV is relatively unimportant. The relaxation time of the polymer is found by initially stretching the polymer to 99\% of its maximum length and allowing it to relax to equilibrium in the absence of any external flow field. The largest relaxation time for the polymer is then found by fitting the tail of the end-to-end distance versus time curve to an exponential function. Relaxation times for the various models are listed in Table I. For the FD models (No. 1 and No. 4 in Table I), the relaxation times are lower than the Rouse relaxation time, the characteristic relaxation time for a chain with no HI or EV and Hookean springs. This difference is due to our method of determining relaxation times. We used the range of polymer extensions from $10 \%$ to $1 \%$ of full extension for the determination of relaxation times in all models. If the relaxation time is calculated for polymer extensions closer to the equilibrium end-to-end distance, or, equivalently, at longer times in the relaxation simulation, the relaxation time constants for the FD models approach that of the Rouse model.

While these polymer models incorporate the large spectrum of time scales, HI, and EV that are present in real polymers, they would be very computationally expensive to use in the coupled flow calculations. For the coupled calculations, ${ }^{10,11}$ we calculate the polymer stress using another idealized polymer model, the FENE-P dumbbell model, ${ }^{12}$

$$
\begin{gathered}
\frac{\boldsymbol{\alpha}}{1-\frac{\operatorname{tr} \boldsymbol{\alpha}}{b}}+\operatorname{We}\left(\frac{\partial \boldsymbol{\alpha}}{\partial t}+(\mathbf{v} \cdot \boldsymbol{\nabla} \boldsymbol{\alpha})-\{\boldsymbol{\alpha} \cdot \boldsymbol{\nabla} \mathbf{v}\}-\{\boldsymbol{\alpha} \cdot \boldsymbol{\nabla} \mathbf{v}\}^{T}\right) \\
=\left(\frac{b}{b+2}\right) \boldsymbol{\delta}, \\
\boldsymbol{\tau}_{p}=n k_{\mathrm{B}} T \frac{b+5}{b}\left[\frac{\boldsymbol{\alpha}}{1-\frac{\operatorname{tr} \boldsymbol{\alpha}}{b}}-\left(1-\frac{2}{b+2}\right) \boldsymbol{\delta}\right],
\end{gathered}
$$

where $\boldsymbol{\alpha}=H\langle\mathbf{q q}\rangle / k_{\mathrm{B}} T$ is the nondimensional structure tensor and $b$ is proportional to the maximum extension of the dumbbell- $\operatorname{tr} \boldsymbol{\alpha}$ cannot exceed $b$. To relate the FENE-P dumbbell model to the bead-spring-chain models, we note that $b=N_{s} b_{s}=H Q_{0}^{2} / k_{\mathrm{B}} T=3 N_{s} N_{k, s}$.

The flow field to which the polymer chains are subjected is that described and pictured in the Introduction. The parameters for the flow are $\mathrm{We}=\lambda U / L$, where $U / L$ is the nominal shear rate (i.e., the shear rate at the wall) for the model flow, and $\mathrm{We}_{\sigma}=\lambda \sigma_{\max }$, the Weissenberg number based on the largest Lyapunov exponent for the model flow, which is $\sigma_{\max }=0.030$.

\section{RESULTS}

As stated previously, the Weissenberg number based on the largest Lyapunov exponent for a flow, $\mathrm{We}_{\sigma}=\lambda \sigma_{\max }$, must be $>\frac{1}{2}$ for a Hookean dumbbell model of a polymer to stretch indefinitely in flow. We begin the presentation of results by showing that this criterion on $\mathrm{We}_{\sigma}$ also applies to the more complicated bead-spring models. Figure 2(a) shows ensemble averages of polymer extensions at four different Weissenberg numbers for model 2 in Table I. Starting from an equilibrium polymer configuration at $t=0$ (time is scaled by the characteristic time for the flow, $L / U$ ), the polymers are stretched as they move along a fluid trajectory in the ECS. As $\mathrm{We}_{\sigma}$ increases to 0.5 and above, the transient polymer extension increases dramatically. The effect of changing the ensemble size is also shown in Fig. 2(b), which is the same as Fig. 2(a) but averages are for an ensemble of 100 bead-spring chains instead of 50 . Very little difference is evident in the averages, except at the lowest We, where the maximum stretch for the smaller ensemble is slightly greater than for the larger ensemble

Since it is known that the appearance of large polymer stretch depends on the largest Lyapunov exponent and that the ECS flows and simple, steady, homogeneous, extensional flows both have positive Lyapunov exponents, a comparison of polymer stretch in the two flows is warranted. Figure 3 shows the extension of a polymer in the ECS flow and in uniaxial extension versus $\mathrm{We}_{\sigma}$. The ECS data of Fig. 3 are the time averages and maximum values of the data series presented in part in Fig. 2. In the case of uniaxial extension the largest Lyapunov exponent for the flow is simply the elongation rate, $\dot{\varepsilon}$, so the quantity $\lambda \dot{\varepsilon}$ is equivalent to $\mathrm{We}_{\sigma}$. The time-averaged polymer extension in the ECS and the steady-state extension in uniaxial elongation become large only for $\mathrm{We}_{\sigma} \gtrsim 1 / 2$. Also shown in Fig. 3 is the maximum extension of the polymer in the ECS. This quantity becomes large as $\mathrm{We}_{\sigma}$ approaches $1 / 2$, so the criterion, which is based on a time-integrated quantity, the Lyapunov exponent, is seen to be relevant for instantaneous as well as average stretch. This result is again seen when using the simpler FENE-P continuum model in the ECS.

A note of caution is warranted at this point in the discussion. In a real drag-reducing polymer solution, the reaction of the polymer on the flow is likely to modify the local flow 

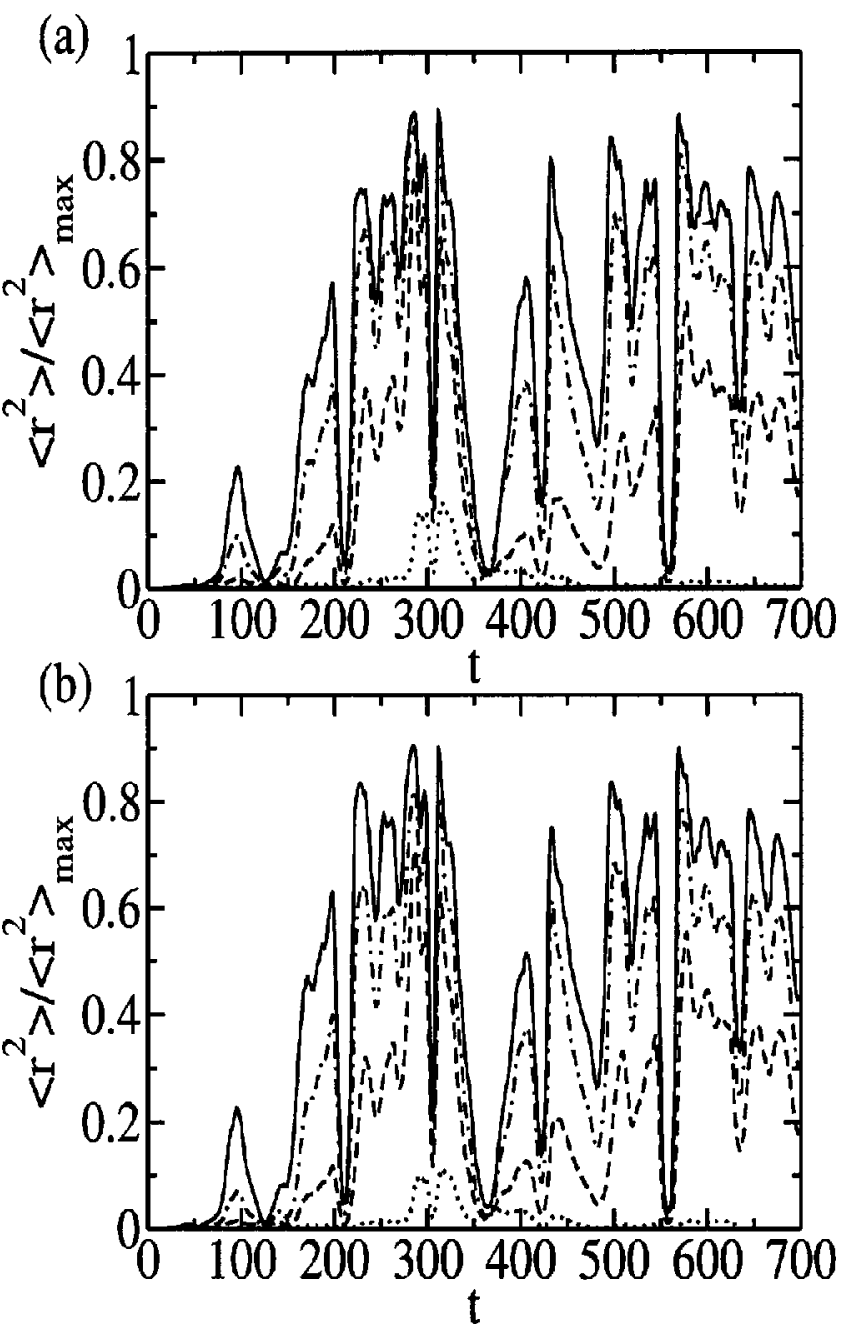

FIG. 2. Average polymer stretch versus time for increasing values of $\mathrm{We}_{\sigma}$ $\ldots \mathrm{We}^{2}=11 \quad\left(\mathrm{We}_{\sigma}=0.34\right) ;---, \mathrm{We}=17 \quad\left(\mathrm{We}_{\sigma}=0.51\right) ;-\cdot-\mathrm{We}=26$ $\left(\mathrm{We}_{\sigma}=0.79\right) ;-, \mathrm{We}=40 \quad\left(\mathrm{We}_{\sigma}=1.21\right)$. Time is scaled by $U / L$. $\left\langle r^{2}\right\rangle /\left\langle r^{2}\right\rangle_{\max }$ is the ensemble average of the square of the end-to-end length divided by the square of the maximum chain length. These results are for $\mathrm{HI} / \mathrm{EV}$ chains-model 2 in Table I. (a) Averages for an ensemble of 50 chains. (b) Averages for an ensemble of 100 chains.

field before the polymer nears full extension. We see this as well in our viscoelastic ECS results. ${ }^{11}$ In that study, as we increase the contour length of our polymers, which increases the drag reduction at a given We, the ratio of the maximum stretch to the contour length decreases compared to shorter polymers. This indicates that the flow field is modified by the polymers so that it is not as effective in stretching the polymers.

To further compare the FENE chains to FENE-P dumbbells, we now observe how the transient extension for the FENE-P model compares to that of free-draining chain models and ones with HI and EV. Figures 4 and 5, like Fig. 2, show the ensemble average extension for different polymer models in the ECS, for $\mathrm{We}_{\sigma}=0.51$ and 1.2. The FENE-P model overpredicts the stretch at some of the higher extensions but through most of the flow field it matches well with the more detailed models. This overprediction of extension is well known in the FENE-P model. ${ }^{47}$ The FENE-P spring force is given by

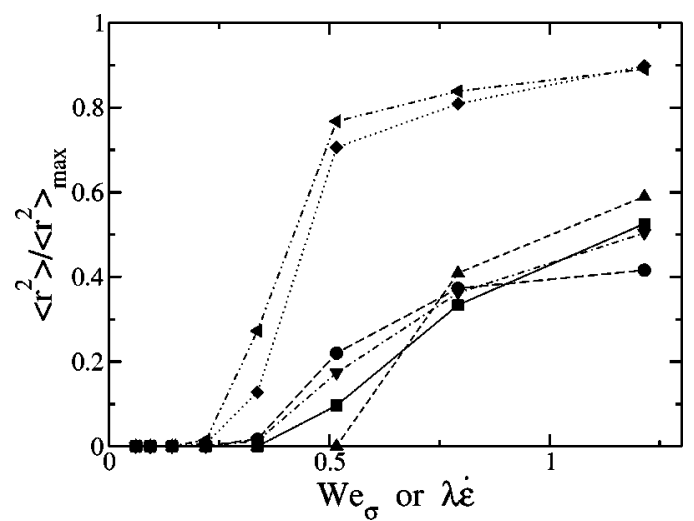

FIG. 3. A comparison of extension in uniaxial extension and ECS flows $(b=117300)$. Averages for the ECS flow are taken over at least 100 polymer relaxation times. Uniaxial extension: $\mathbf{\square}-$ FD FENE chain (model 1 in Table I), $\mathbf{\Delta}-\mathrm{HI} / \mathrm{EV}$ FENE chain (model 2). Time-averaged extension in the ECS: $\boldsymbol{\nabla}$-FD FENE chain (model 1), - - HI/EV FENE chain (model 2). Maximum extension in the ECS: 4-FD FENE chain (model 1), $\checkmark-\mathrm{HI} / \mathrm{EV}$ FENE chain (model 2).

$$
\mathbf{F}^{(c)}=\frac{H \mathbf{Q}}{1-\left\langle Q^{2} / Q_{0}^{2}\right\rangle} ;
$$

hence, the FENE-P model only dictates that the ensemble average of the stretch be less than the maximum extension, which leads to the discrepancy. The effects of HI and EV are also seen in Fig. 4. The free-draining model shows consistently lower stretch than both the HI/EV FENE chains and FENE-P demonstrating that the polymer-solvent interactions have an effect on the polymer extension in complex flows. With HI, the cooperative motion among the beads tends to speed relaxation to equilibrium relative to a free-draining model. Therefore, to match relaxation times between the models, the beads of a HI/EV model must be given a higher friction coefficient than that of the FD model. When the $\mathrm{HI} / \mathrm{EV}$ model becomes highly stretched, the beads are far apart, causing the velocity perturbations to be negligible. So at large extensions, our HI/EV models behave essentially like a FD model but with a greater bead drag than the true FD

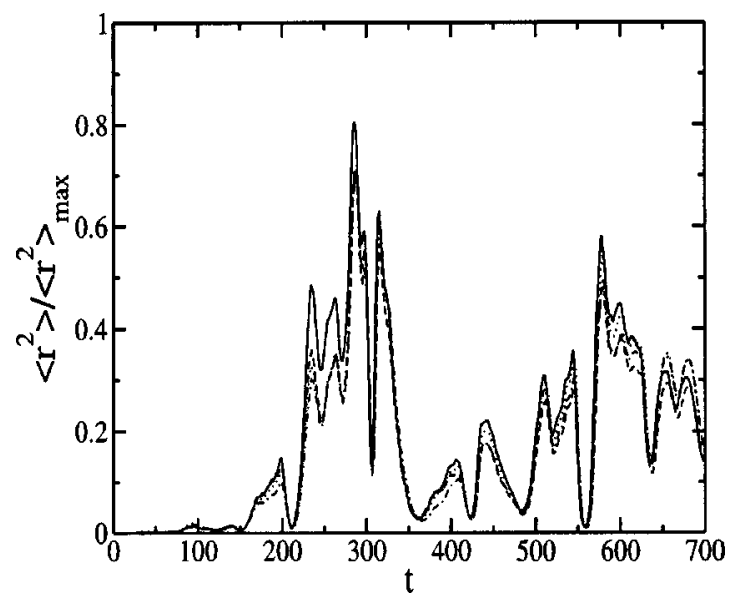

FIG. 4. A comparison of extension for FENE chains and FENE-P, $\mathrm{We}_{\sigma}$ $=0.51$. - , FENE-P; ---, FD FENE chain (model 1 in Table I), - - , $\mathrm{HI} / \mathrm{EV}$ chain $-v=1($ model 2$), \cdots, \mathrm{HI} / \mathrm{EV}$ chain $-v=20$ (model 3$)$. 
(a)
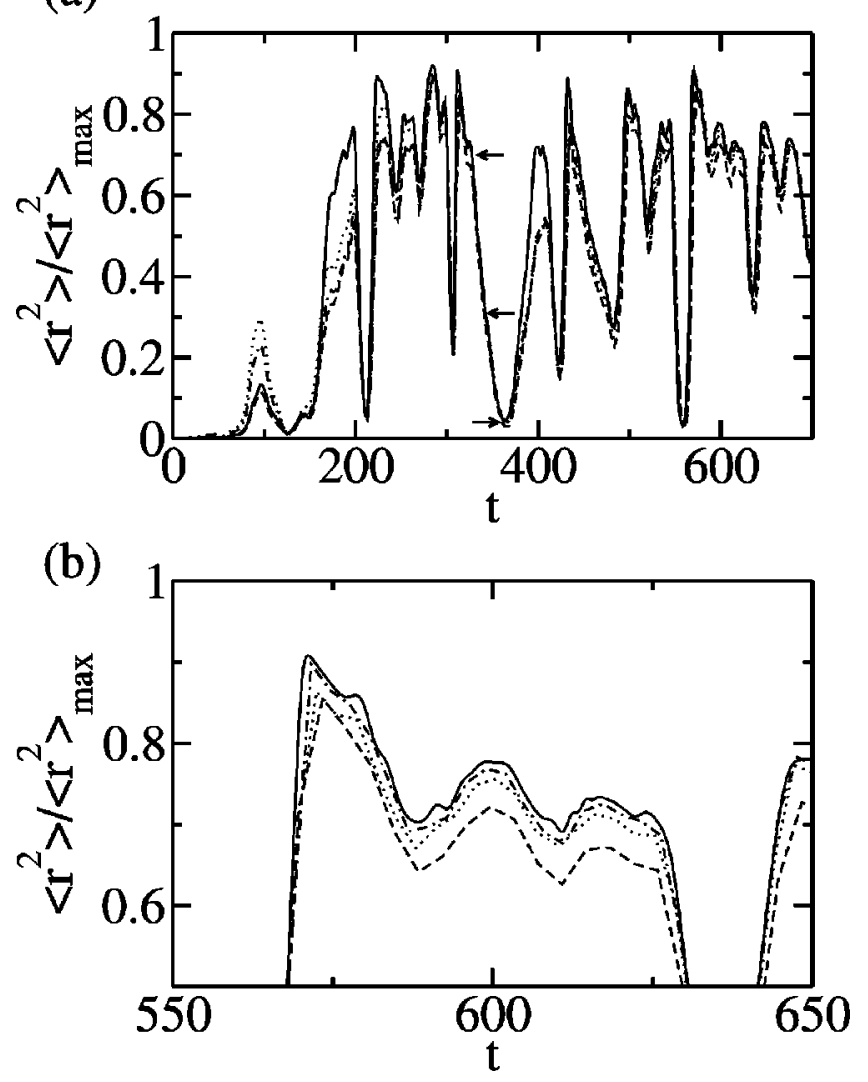

FIG. 5. A comparison of the extension for FENE chains and FENE-P, $\mathrm{We}_{\sigma}=1.2$. - FENE-P; ---, FD FENE chain (model 1 in Table I), - - HI/EV chain $-v=1(\operatorname{model} 2), \cdots, \mathrm{HI} / \mathrm{EV}$ chain $-v=20(\operatorname{model} 3)$. Plot (b) is a blowup of the behavior in part (a) for $550<t<650$. The arrows in plot (a) indicate where the PDF's in Fig. 6 are in the trajectory.

model. Since the beads of the HI/EV chain experience higher drag as the chain becomes extended, the flow is able to stretch them more than it does the FD chain.

The probability density function (PDF) for the stretch of the ensemble of chains for three times as the polymer relaxes are given in Fig. 6. The PDFs at the times shown are steep,

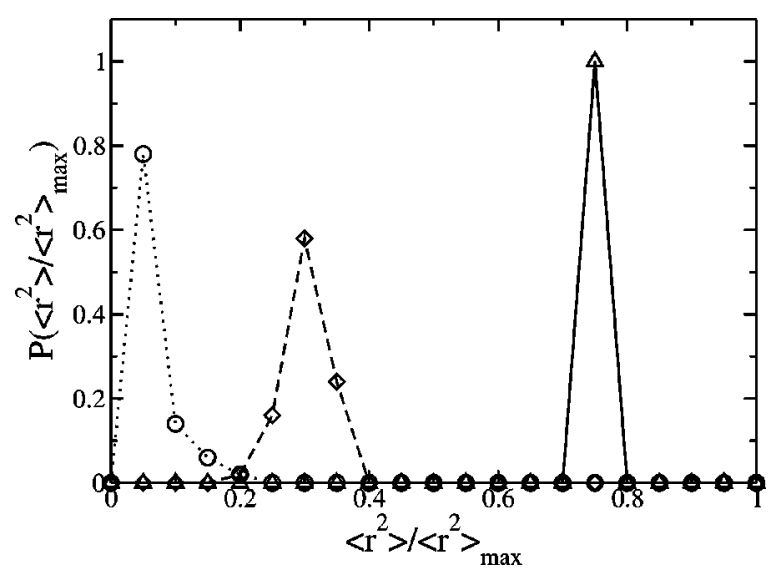

FIG. 6. Probability density function (PDF) for the polymer stretch. The PDF for the ensemble of FENE HI/EV chains (model 2 in Table I) is shown for three different times: $\triangle-t=325.27, \diamond-t=344.50, \bigcirc-t=364.30$. These times are indicated by arrows in Fig. 5 . The line segments connecting the points are simply to guide the eyes. (a)
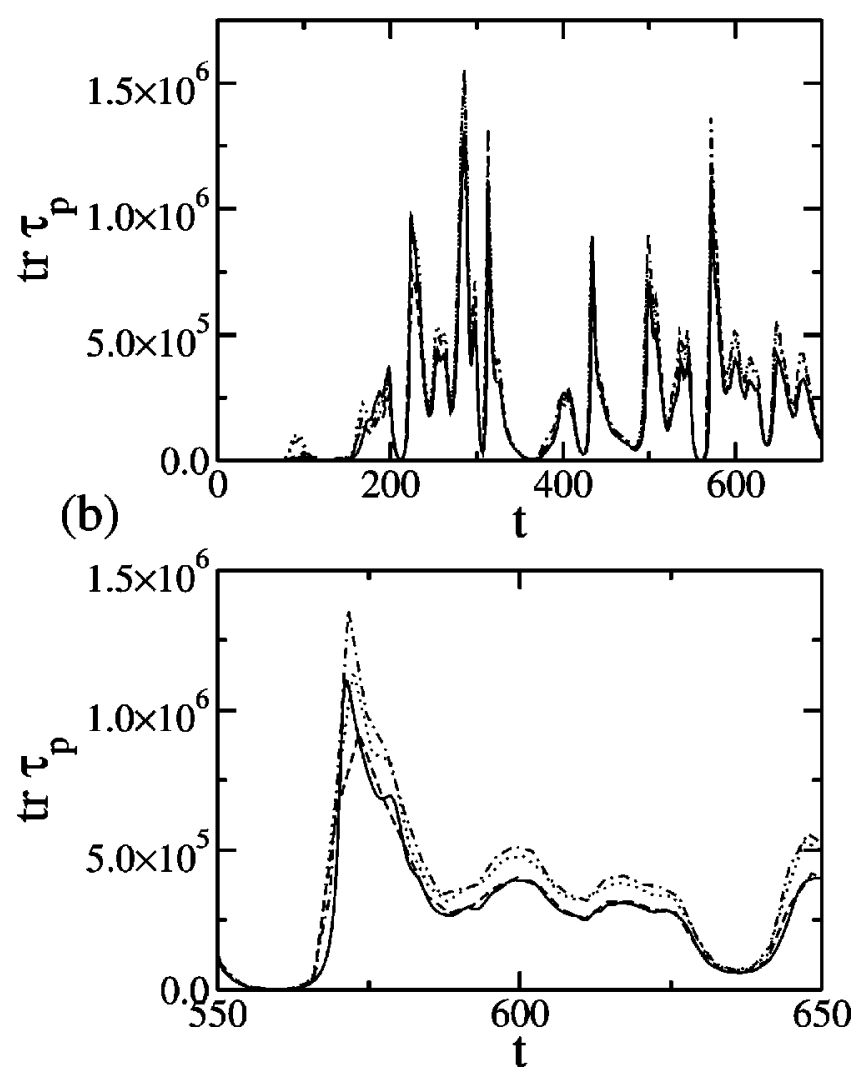

FIG. 7. (a) A comparison of $\operatorname{tr} \tau_{p}$ for FENE chains and FENE-P, We $\mathrm{W}_{\sigma}$ 1.2. - FENE-P; --- FD FENE chain (model 1 in Table I); - - - , HI/EV chain $-v=1$ (model 2), $\cdots$, HI/EV chain $-v=20$ (model 3). Part (b) is a blowup of the behavior in (a) for $550<t<650$.

single peaks. Although not shown here, the PDFs throughout the trajectory tend to show narrow, single peaks. This result is not surprising because the Brownian force is the only difference among the chains in the ensemble. In a strong flow $\left(\mathrm{We}_{\sigma}>\frac{1}{2}\right.$ ), the hydrodynamic drag forces on the beads are dominant. Since the drag forces are the same on all chains in the ensemble, only a small spread in the stretch for the ensemble would be expected.

Figure 7 shows the same result as Fig. 5, but in terms of the trace of the polymer stress tensor calculated for the different models. Note that a dramatic temporal variation in the stress level is found - this variation translates into large spatial inhomogeneities in stress. The FENE-P prediction of the stress is generally smaller than but close to the more detailed models; we elaborate on this observation below. It is also seen that the addition of $\mathrm{HI}$ and $\mathrm{EV}$ cause the stress to be slightly greater in this model compared to the free-draining model. The most important observation that can be drawn from this plot, however, is that even when FENE-P is not quantitatively accurate, it tracks very faithfully the timedependence of the stresses in the more detailed modelswhere the FENE-P stress is large, so is that for the chain. This result implies that the FENE-P model will faithfully capture the spatial distribution of stress in the ECS flow. This favorable result suggests that the use of the relatively simple continuum model in the coupled calculations will 


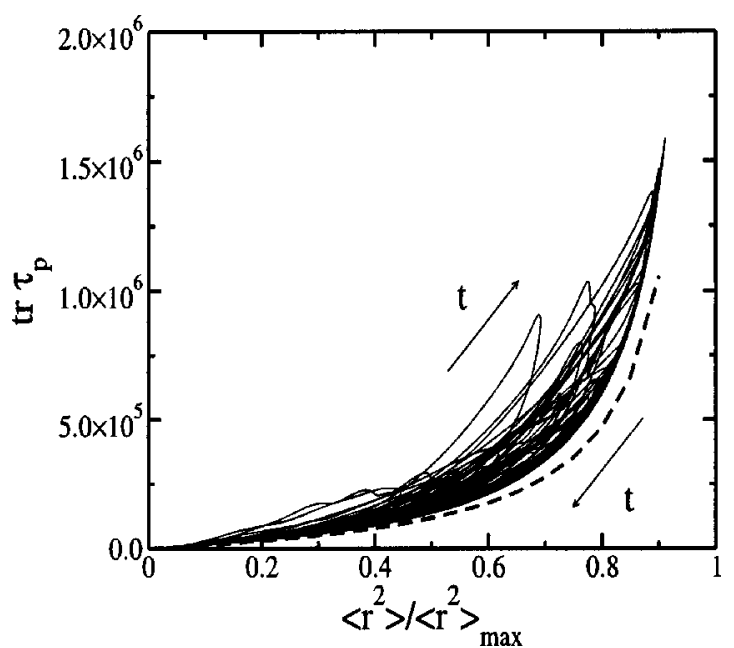

FIG. 8. Polymer stress versus stretch for a bead-spring model and for FENE-P, $b=117300$. - , FENE chain with HI/EV (model 2 in Table I), --- , FENE-P. The direction of time is clockwise for the HI/EV data.

yield reasonable predictions of how the ECS are affected by viscoelasticity.

To further elaborate on this point, we note that one of the known deficiencies of the FENE-P model is that FENE-P does not show "stress hysteresis." 48,49 For real polymers two conformations with the same end-to-end distance can have different internal configurations, leading to different values of stress. In the FENE-P model, the stress depends only upon the current end-to-end distance so this hysteresis is not present. Figure 8 shows the stress versus extension for a chain model and for the FENE-P model moving along a fluid trajectory in the ECS. The chain model clearly shows hysteresis. The direction of time around the hysteresis loop is clockwise, so the highest stress for a given end-to-end distance occurs as the polymer is being stretched. In the relaxation portion of the curve, the polymer stress for the FENE chain is still higher than the FENE-P dumbbell, although there is less of a difference than in the stretching portion of

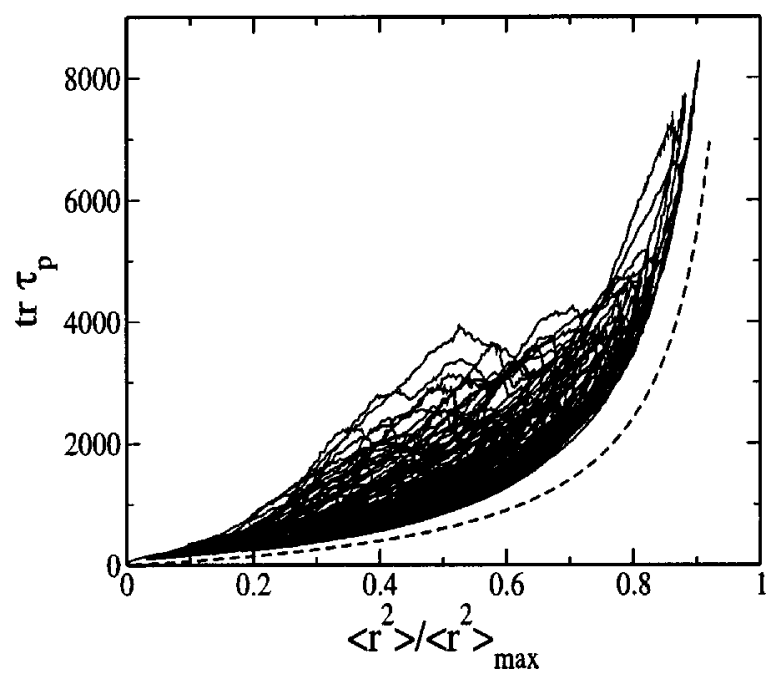

FIG. 9. Polymer stress versus stretch for a chain bead-spring model and for FENE-P, $b=600$. - , FENE chain with HI/EV (model 5 in Table I); ---, FENE-P. The direction of time is clockwise for the HI/EV data.

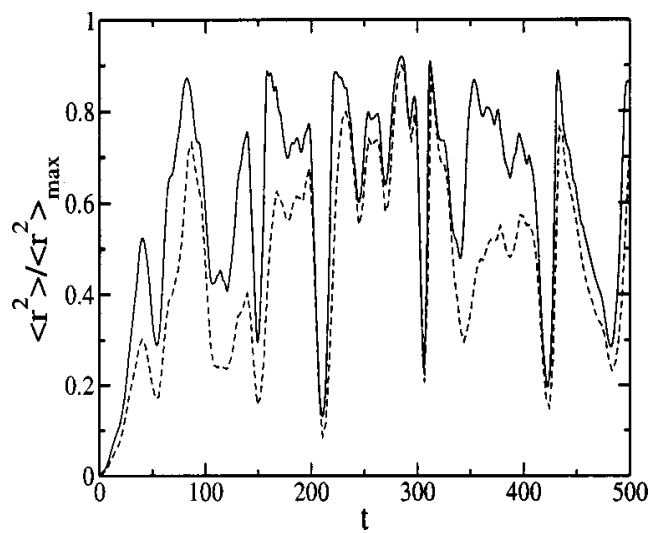

FIG. 10. A comparison of extension for FENE chains and FENE-P, We $\mathrm{W}_{\sigma}$ $=1.2$ and $b=600$. - , FENE-P,,--- FD FENE chain (model 4 in Table I).

the curve. (We are using the term "relaxation" to describe the decrease in the end-to-end distance of the polymers, but recall that the polymers are in the ECS flow field and not relaxing in the absence of an external flow.) Recalling the result of the coupled calculations mentioned in the Introduction, the streamwise vortices are weakened by the relaxation of the polymer as it leaves the streak. Figure 9 shows that the polymer stress here is slightly higher than that predicted by the FENE-P dumbbell model and may have a greater effect on the ECS than the coupled calculations suggest. But, as shown earlier, the FENE-P qualitatively agrees with the more complicated bead-spring models. Future work will determine the importance of the stress hysteresis in flows with complex kinematics.

The above results show that the FENE-P dumbbell does reasonably well at the long contour lengths typical of real drag-reducing polymers. In fully coupled calculations, however, the maximum length used is much smaller than that considered above. ${ }^{11,13,14,50}$ Figures 10 and 11 compare the extension and stress for a model of a much smaller polymer, with $b=600$. For this value of $b$, the overprediction of the extension by the FENE-P model is more pronounced than in the case of large $b$. However, the stress given by the FENE-P model is still reasonably close to that given by the more detailed bead-spring model. The agreement between the two

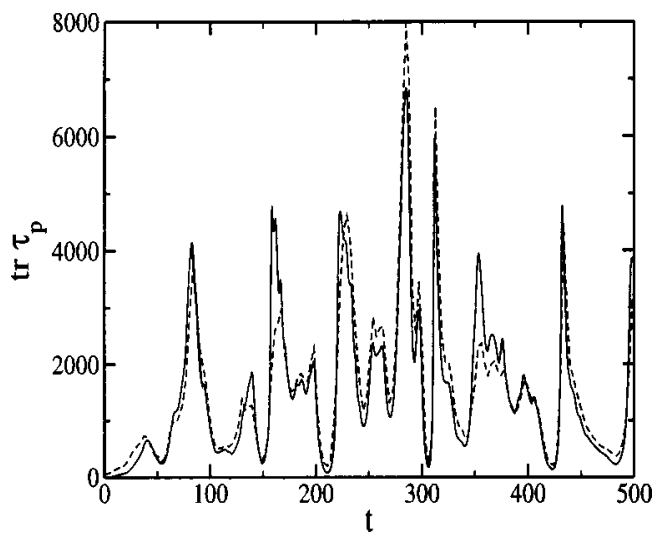

FIG. 11. A comparison of tr $\tau_{p}$ for FENE chains and FENE-P, $\mathrm{We}_{\sigma}=1.2$ and $b=600$. - , FENE-P, --- FD FENE chain (model 4 in Table I). 

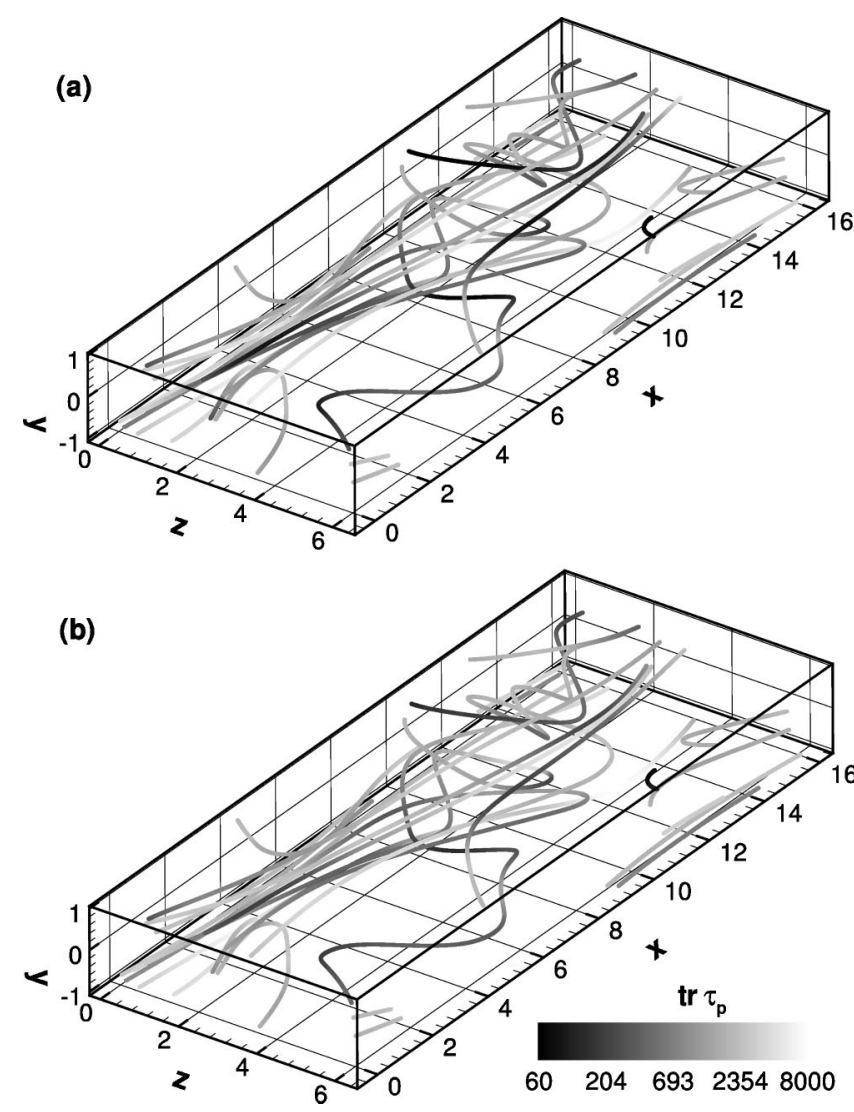

FIG. 12. Polymer stress for (a) FENE-P dumbbell and (b) FENE chain along the same trajectory, $\mathrm{We}_{\sigma}=1.2$ and $b=600$.

models is also illustrated in Fig. 12, which shows the polymer stress for the FENE-P and FENE chain models along the same fluid trajectory as a function of spatial position in the flow field. (Recall that although the paths of fluid elements are chaotic, the velocity field itself is steady.) As argued above based on the time-evolution plots, we see here that large stresses occur in the flow at the same spatial positions for the FENE-P and more detailed models. The highest polymer stress is seen to occur in the streamwise streak. The polymer stretch decreases as polymer leaves the streak and moves into and around one of the vortices. This relaxation of the polymer tends to reduce the strength of the vortices, which causes the self-sustaining process to collapse and leads to drag reduction. ${ }^{10,11}$

\section{CONCLUSIONS}

We performed Brownian dynamics (BD) simulations of passive bead-spring chains in a model of the turbulent buffer region to determine the behavior of polymers in turbulence and to evaluate the use of the FENE-P dumbbell model in coupled calculations. The simulations were performed along trajectories in the flow field of an "exact coherent state" (ECS), one of a recently discovered class of nontrivial threedimensional flow patterns in the plane Couette geometry. The ECS provide a valuable model for studying the behavior of polymers in shear turbulence because they capture the dominant structure of the buffer region: streamwise streaks flanked by pairs of counter-rotating, streamwise vortices.
Streamlines in the ECS are chaotic and, equivalently, the largest Lyapunov exponent for the flow field is positive. For all flows with chaotic streamlines, which includes many, if not most, three-dimensional, incompressible flows, polymers in the flow become highly stretched only when the product of the longest polymer relaxation time and the largest Lyapunov exponent for the velocity field, $\mathrm{We}_{\sigma}=\lambda \sigma_{\max } \gtrsim \frac{1}{2}$. This result unifies the previous results for polymers in various flows with strong elongational components. Although the onset value of $\mathrm{We}_{\sigma}=\frac{1}{2}$ is only rigorous for a Hookean dumbbell, both large instantaneous and time-averaged stretch are seen for more complicated bead-spring models when this criterion is exceeded.

For both bead-spring chain and dumbbell models, the kinematics of the ECS cause large variations in the polymer extension and stress. The addition of hydrodynamic interactions (HI) and excluded volume (EV) to the bead-spring model has no qualitative effect on the polymer stretch once the results are renormalized for the change in the longest relaxation time of the model. Since the flow field causes the polymers to be stretched far from equilibrium, the interactions among beads through $\mathrm{HI}$ and EV are small. Therefore, not much difference is seen between $\mathrm{HI} / \mathrm{EV}$ and freedraining (FD) models.

The FENE-P model, which is used in many calculations where the polymer stress is coupled to the flow field, is found to qualitatively capture the features of the bead-spring models in a complex flow field. For both long polymers (comparable to those used in experiment) and short polymers (similar in size to those used in the coupled flow calculations), the FENE-P compares well in terms of polymer stretch and stress to the FENE chains. FENE-P slightly overpredicts stretch in areas where velocity gradients are high and polymer stretch is large. This is consistent with the findings of Ilg et al. ${ }^{38}$ in a comparison of FENE-P and FENE dumbbells in a wall turbulent flow taken from DNS. Most importantly, the FENE-P model shows large stress for the same spatial positions as the FENE chain. For both models, the majority of the stretching takes place as the polymer moves through the streamwise streaks. The polymer relaxes as it leaves the streak and enters a vortex. The relaxation of the polymer as it enters the vortex generates a force due to the polymer (proportional to the divergence of the polymer stress, $\boldsymbol{\nabla} \cdot \boldsymbol{\tau}_{p}$ ) that is negatively correlated with the $x$ - and $y$-velocity fluctuations. ${ }^{11}$ This force due to the polymer weakens the vortex and leads to a collapse of the selfsustaining process that underlies the structures of the buffer region. Due to the absence of stress hysteresis in the FENE-P model, it underpredicts the stress during relaxation. Therefore we expect that this model will somewhat underpredict the changes in turbulent structure brought on by polymers. However, since the overall structure of the stress field is the same for the FENE-P and full-chain models, it seems likely that the absence of stress hysteresis in FENE-P will not change the overall mechanistic picture of drag reduction that is emerging from FENE-P-based computational studies of the ECS and of fully developed turbulence. 


\section{ACKNOWLEDGMENTS}

The authors wish to thank Fabian Waleffe for the use of his ECS codes and Richard M. Jendrejack for his help in implementing the Brownian dynamics simulations. We also gratefully acknowledge support from the National Science Foundation (NSF) and the donors of the Petroleum Research Fund, administered by the American Chemical Society.

${ }^{1}$ J. L. Lumley, "Drag reduction by additives," Annu. Rev. Fluid Mech. 1, 367 (1969).

${ }^{2}$ W. D. McComb, The Physics of Fluid Turbulence (Oxford University Press, New York, 1990)

${ }^{3}$ P. S. Virk, "Drag reduction fundamentals," AIChE J. 21, 225 (1975).

${ }^{4}$ S. K. Robinson, "Coherent motions in the turbulent boundary layer," Annu. Rev. Fluid Mech. 23, 601 (1991).

${ }^{5}$ J. M. J. den Toonder, M. A. Hulsen, G. D. C. Kuiken, and F. T. M. Nieuwstadt, "Drag reduction by polymer additives in a turbulent pipe flow: Numerical and laboratory experiments," J. Fluid Mech. 337, 193 (1997).

${ }^{6}$ G. L. Donohue, W. G. Tiederman, and M. M. Reischman, "Flow visualization of the near-wall region in a drag-reducing channel flow," J. Fluid Mech. 50, 559 (1972).

${ }^{7}$ A. A. Draad, G. D. C. Kuiken, and F. T. M. Nieuwstadt, "Laminarturbulent transition in pipe flow for Newtonian and non-Newtonian fluids," J. Fluid Mech. 377, 267 (1998).

${ }^{8}$ M. P. Escudier, F. Presti, and S. Smith, "Drag reduction in the turbulent pipe flow of polymers," J. Non-Newtonian Fluid Mech. 81, 197 (1999).

${ }^{9}$ D. T. Walker and W. G. Tiederman, "Turbulent structure in a channel flow with polymer injection at the wall," J. Fluid Mech. 218, 377 (1990).

${ }^{10}$ P. A. Stone, Master's thesis, University of Wisconsin-Madison, 2002.

${ }^{11}$ P. A. Stone, F. Waleffe, and M. D. Graham, "Toward a structural understanding of turbulent drag reduction: Nonlinear coherent states in viscoelastic shear flows," Phys. Rev. Lett. 89, 208301 (2002)

${ }^{12}$ R. B. Bird, C. F. Curtiss, R. C. Armstrong, and O. Hassager, Dynamics of Polymeric Liquids, 2nd ed. (Wiley, New York, 1987), Vol. 2.

${ }^{13}$ C. D. Dimitropoulos, R. Sureshkumar, A. N. Beris, and R. A. Handler, "Budgets of Reynolds stress, kinetic energy and streamwise enstrophy in viscoelastic turbulent channel flow," Phys. Fluids 13, 1016 (2001).

${ }^{14}$ R. Sureshkumar, A. N. Beris, and R. Handler, "Direct numerical simulation of the turbulent channel flow of a polymer solution," Phys. Fluids 9, 743 (1997)

${ }^{15}$ W. G. Tiederman, T. S. Luchik, and D. G. Bogard, "Wall-layer structure and drag reduction," J. Fluid Mech. 156, 419 (1985).

${ }^{16}$ A. J. Chorin, Vorticity and Turbulence (Springer-Verlag, New York, 1994).

${ }^{17}$ R. M. Clever and F. H. Busse, "Tertiary and quaternary solutions for plane Couette flow," J. Fluid Mech. 344, 137 (1997).

${ }^{18}$ M. Nagata, "Bifurcation in Couette flow between almost corotating cylinders," J. Fluid Mech. 169, 229 (1986).

${ }^{19}$ M. Nagata, "On wavy instabilities of the Taylor-vortex flow between corotating cylinders," J. Fluid Mech. 188, 585 (1988).

${ }^{20} \mathrm{M}$. Nagata, "Three-dimensional finite amplitude solutions in plane Couette flow: Bifurcation from infinity," J. Fluid Mech. 217, 519 (1990).

${ }^{21} \mathrm{~F}$. Waleffe, "On a self-sustaining process in shear flows," Phys. Fluids $\mathbf{9}$, 883 (1997)

${ }^{22} \mathrm{~F}$. Waleffe, "Three-dimensional coherent states in plane shear flows," Phys. Rev. Lett. 81, 4140 (1998).

${ }^{23} \mathrm{~F}$. Waleffe, "Exact coherent structures in channel flow," J. Fluid Mech. 435, 93 (2001).

${ }^{24} \mathrm{~A}$ saddle-node bifurcation, also known as a limit point or fold, arises when two steady states appear spontaneously as a parameter increases.
${ }^{25}$ S. H. Strogatz, Nonlinear Dynamics and Chaos: With Applications to Physics, Biology, Chemistry and Engineering (Addison-Wesley, New York, 1994).

${ }^{26}$ S. Bottin, O. Dauchot, F. Daviaud, and P. Mannveille, "Experimental evidence of streamwise vorticies as finite amplitude solutions in transitional plane Couette flow," Phys. Fluids 10, 2597 (1998).

${ }^{27}$ F. Daviaud, J. Hegseth, and P. Bergé, "Subcritical transition to turbulence in plane Couette flow," Phys. Rev. Lett. 69, 2511 (1992).

${ }^{28} \mathrm{~F}$. Waleffe, "Homotopy of exact coherent structures in plane shear flows," Phys. Fluids (to be published).

${ }^{29}$ J. Jimenez and M. P. Simens, "Low-dimensional dynamics of a turbulent wall flow," J. Fluid Mech. 435, 81 (2001).

${ }^{30}$ G. Kawahara and S. Kida, "Periodic motion embedded in plane Couette turbulence: Regeneration cycle and burst," J. Fluid Mech. 449, 291 (2001).

${ }^{31}$ J. L. Lumley, "On the solution of equations describing small scale deformation," Symp. Math. 9, 315 (1972).

${ }^{32}$ E. Balkovsky, A. Fouxon, and V. Lebedev, "Turbulent dynamics of polymer solutions," Phys. Rev. Lett. 84, 4765 (2000).

${ }^{33}$ M. Chertkov, "Polymer stretching by turbulence," Phys. Rev. Lett. 84, 4761 (2000).

${ }^{34}$ L. G. Leal, "Dynamics of dilute polymer solutions," in Structure of Turbulence and Drag Reduction (Springer-Verlag, Berlin, 1990).

${ }^{35}$ H. Massah, K. Kontomaris, W. R. Schowalter, and T. J. Hanratty, "The configurations of a FENE bead-spring chain in transient rheological flows and in a turbulent flow," Phys. Fluids A 5, 881 (1993).

${ }^{36} \mathrm{H}$. Massah and T. J. Hanratty, "Added stresses because of the presence of FENE-P bead-spring chains in a random velocity field," J. Fluid Mech. 337, 67 (1997).

${ }^{37}$ A. B. Mosler and E. S. G. Shaqfeh, "The conformation change of model polymers in stochastic flow fields: Flow through fixed beds," Phys. Fluids 9, 1222 (1997).

${ }^{38}$ P. Ilg, E. De Angelis, I. V. Karlin, C. M. Casciola, and S. Succi, "Polymer dynamics in wall turbulent flow," Europhys. Lett. 58, 616 (2002).

${ }^{39}$ H. C. Öttinger, Stochastic Processes in Polymeric Fluids (Springer-Verlag, New York, 1996).

${ }^{40}$ R. B. Bird, C. F. Curtiss, R. C. Armstrong, and O. Hassager, Dynamics of Polymeric Liquids, 2nd ed. (Wiley, New York, 1987), Vol. 1.

${ }^{41}$ E. Balkovsky, A. Fouxon, and V. Lebedev, "Turbulence of polymer solutions," Phys. Rev. E 64, 056301 (2001).

${ }^{42}$ S. S. Girimaji and S. B. Pope, "Material-element deformation in isotropic turbulence," J. Fluid Mech. 220, 427 (1990).

${ }^{43}$ R. Jendrejack, J. J. de Pablo, and M. D. Graham, "Stochastic simulations of DNA in flow: Dynamics and the effects of hydrodynamic interactions," J. Chem. Phys. 116, 7752 (2002).

${ }^{44}$ R. M. Jendrejack, M. D. Graham, and J. J. de Pablo, "Hydrodynamic interactions in long chain polymers: Application of the Chebyshev polynomial approximation in stochastic simulations," J. Chem. Phys. 113, 2894 (2000).

${ }^{45}$ M. Doi and S. F. Edwards, The Theory of Polymer Dynamics (Oxford University Press, New York, 1986).

${ }^{46}$ J. Rotne and S. Prager, "Variational treatment of hydrodynamic interactions in polymers," J. Chem. Phys. 50, 4831 (1969).

${ }^{47}$ M. Herrchen and H. C. Öttinger, "A detailed comparison of various FENE dumbbell models," J. Non-Newtonian Fluid Mech. 68, 17 (1997).

${ }^{48}$ I. Ghosh, G. H. McKinley, R. A. Brown, and R. C. Armstrong, "Deficiencies of FENE dumbbell models in describing the rapid stretching of dilute polymer solutions," J. Rheol. 45, 721 (2001).

${ }^{49}$ R. G. Larson, Constitutive Equations for Polymer Melts and Solutions (Butterworths, Boston, 1988).

${ }^{50}$ S. Sibilla and A. Baron, "Polymer stress statistics in the near-wall turbulent flow of a drag-reducing solution," Phys. Fluids 14, 1123 (2002). 